\title{
A SINR Maximizing RAKE Receiver for DS-CDMA Downlinks
}

\author{
Massimiliano Lenardi Abdelkader Medles Dirk T.M. Slock \\ Institut Eurécom*, 2229 route des Crêtes, B.P. 193, \\ 06904 Sophia Antipolis Cedex, FRANCE \\ Tel: +33 49300 2910/2606; Fax: +3349300 2627 \\ e-mail: $\{$ lenardi, medles, slock\}@eurecom.fr
}

\begin{abstract}
The RAKE receiver is a matched filter $(M F)$, matched to the operations of spreading, pulse shape filtering and channel filtering. An SINR maximizing linear receiver may perform much better. In the downlink, in which the channel is the same for all intracell signals, and with orthogonal codes and cell-dependent scrambling, good SINR performance can be attained with a RAKE-like receiver. In particular, we replace the pulse-shape MF by another filter, and the sparse channel MF by another sparse filter. The first filter is chosen to facilitate the design/adaptation of the sparse filter, the coefficients of which are optimized for maximum SINR. In the presence of intercell interference (other base stations), but using multiple receiver antennas and/or oversampling with respect to the chip rate, good SINR performance can be attained with these structured linear receivers.
\end{abstract}

\section{INTRODUCTION}

In the FDD mode of the Wideband CDMA (WCDMA) option of the 3GPP UMTS proposal for cellular wireless communications, both uplink and downlink use DS-CDMA communications. This paper focuses on the downlink, where a set of orthogonal periodic spreading sequences are used, to take advantage of the synchronicity (between users) of the downlink.

To limit interference between cells though, a celldependent scrambling gets added which does not destroy the orthogonality between the intracell users. Due to the scrambling (which can be considered as a stationary chip

*Eurécom's research is partially supported by its industrial partners: Ascom, Swisscom, Thomson-CSF, IBM France, CEGETEL, Motorola, France Télécom, Hitachi Europe and Texas Instruments. The work leading to this paper was also partially supported by the French RNRT (National Network for Telecommunications Research) project AUBE. rate sequence), the received signal is cyclostationary at the chip rate. The conventional receiver for DS-CDMA communications is the RAKE receiver. The RAKE receiver is a matched filter (MF), matched to the operations of spreading, pulse shape filtering and channel filtering. Such a MF does not maximize the SINR, but only the SNR. The RAKE receiver is a restricted linear optimal receiver in the sense that it would be optimal if only the additive white noise (and not any interference) would be present.

Even though the received signal is cyclostationary at the chip rate (and not at the symbol rate, so that signal subspaces do not exist) linear multiuser detectors (MUD) can be meaningfully applied to achieve much improved performance (SINR) over the RAKE receiver. The general linear MMSE receiver is time-varying however, due to the presence of the scrambler. For such a receiver spanning several symbol periods, the complexity for applying the filter can be quite high, due to the multiplications of signals with arbitrarily valued coefficients at chip rate. And of course, the complexity for producing the time-varying filter coefficients is enormous.

However, structurally constrained linear detectors exist that show a reasonable complexity/performance trade-off. Indeed, apart from synchronicity, another characteristic of the downlink is that all intracell signals pass through the same channel (if the BS does not apply beamforming). So, considering only the intracell interference (and not the intercell interference and noise), a receiver consisting of a zeroforcing equalizer followed by a descrambler and a correlator would be optimal (maximize SIR). Indeed, the equalizer restores orthogonality of the codes (which was destroyed by the delay spread of the multipath channel) so that a simple correlator then suffices to pick out the signal with the code of interest while perfectly suppressing all other (orthogonal) codes. Such a receiver is also suboptimal though since the zero-forcing equalizer enhances the noise and intercell interference. The RAKE maximizes the SNR while this receiver maximizes the SIR (counting the intercell interfer- 
ence with the noise). The performace criterion that needs to be optimized though is the SINR.

In [2] we proposed a generalized linear receiver, the max-SINR receiver, which encompasses the RAKE and the equalizer-plus-correlator receivers [3] as special cases. The structure is the same of the RAKE receiver, but the channel and pulse shape matched filters are replaced by an equalizer filter that is designed to maximize the SINR at the output of the receiver. So the receiver is a cascade of a linear (shortterm) time-invariant equalizer, a descrambler and a correlator. So the overall linear receiver is time-varying but the time-variation is completely concentrated in the descrambler. It turns out that the optimal design of the equalizer that leads to maximum SINR at the output of the overall receiver leads to the MMSE equalizer (with the received signal being cyclostationary at chip rate). In [4] we studied different lower-complexity implementations of the equalizer, including a cascade of a pulse shape matched filter and a sparse filter, whose coefficients were optimized to maximize the output SINR. In this paper we propose to replace the pulse shape matched filter by a pulse shape equalizer, using possibly multiple antennas to better handle intercell interference also.

\section{MULTIUSER DOWNLINK SIGNAL MODEL}

Fig. 1 shows the downlink signal model in baseband, between the mobile receiver and the main base station. Signals coming from other BSs are of the same structure, but passing through different channels, and included in the additive noise $v(t)$. The $K$ users are assumed to transmit linearly modulated signals over the same linear multipath channel. The symbol and chip periods $T$ and $T_{c}$ are related through the spreading factor $L: T=L T_{c}$, which is assumed here to be common for all the users. The total chip sequence $b_{l}$ is the sum of the chip sequences of all the users, each one given by the product between the $n$th symbol of the $k$ th user and an aperiodic spreading sequence $w_{k, l}$ which is itself the product of a periodic Walsh-Hadamard (with unit energy) spreading sequence $\boldsymbol{c}_{k}=\left[\boldsymbol{c}_{k, 0} \boldsymbol{c}_{k, 1} \cdots \boldsymbol{c}_{k, L-1}\right]^{T}$, and a base-station specific unit magnitude complex scrambling sequence $s_{l}$ with variance $1, w_{k, l}=c_{k, l \bmod L} s_{l}$ :

$$
b_{l}=\sum_{k=1}^{K} b_{k, l}=\sum_{k=1}^{K} a_{k,\left\lfloor\frac{l}{L}\right\rfloor} w_{k, l}
$$

The chip sequence $b_{l}$ gets transformed into a continuoustime signal by filtering it with the pulse shape $p(t)$ and then passes through the multipath propagation channel $h(t)$ to yield the received signal $y(t)$. The receiver samples $M$ times per chip the lowpass filtered received signal.

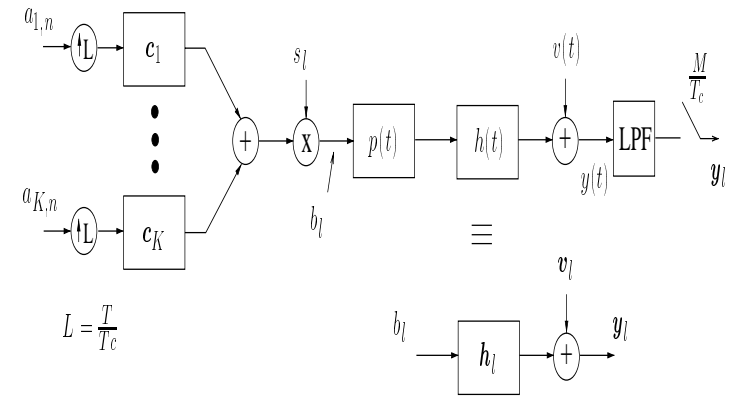

Figure 1. Downlink signal model BS-MS

Stacking the $M$ samples per chip period in vectors, we get for the sampled received signal at the MS antenna $j$

$$
\boldsymbol{y}_{l}^{j}=\sum_{k=1}^{K} \sum_{i=0}^{N-1} \boldsymbol{h}_{i}^{j} b_{k, l-i}+\boldsymbol{v}_{l}^{j},
$$

where

$$
\boldsymbol{y}_{l}^{j}=\left[\begin{array}{c}
y_{1, l}^{j} \\
\vdots \\
y_{M, l}^{j}
\end{array}\right], \boldsymbol{h}_{l}^{j}=\left[\begin{array}{c}
h_{1, l}^{j} \\
\vdots \\
h_{M, l}^{j}
\end{array}\right], \boldsymbol{v}_{l}^{j}=\left[\begin{array}{c}
v_{1, l}^{j} \\
\vdots \\
v_{M, l}^{j}
\end{array}\right]
$$

Here $\boldsymbol{h}_{l}^{j}$ represents the vectorized samples of the overall channel, including pulse shape, propagation channel and receiver filter for the MS antenna $j(j=1 \cdots J)$. The overall channel is assumed to have a delay spread of $N$ chips. In the case of multipath, the channel model is

$$
h_{m, l}^{j}=\sum_{p=1}^{P} \alpha_{p}^{j} p\left(l T_{c}+\frac{(m-1) T_{c}}{M}-\tau_{p}\right)
$$

For antenna $j,\left\{\alpha_{p}^{j}\right\}$ represent the complex amplitudes of the path $p$ with the correspondent delays $\left\{\tau_{p}\right\}$ which are equal for all $P$ MS antennas. If we model the scrambling sequence and the symbol sequences as independent i.i.d. sequences, then the chip sequence $b_{l}$ is a sum of $K$ independent white noises (chip rate i.i.d. sequences, hence stationary). The intracell contribution to $\boldsymbol{y}_{l}^{j}$ then is a stationary (vector) process (the continuous-time counterpart is cyclostationary with chip period). The intercell interference is a sum of contributions that are of the same form as the intracell contribution. The remaining noise is assumed to be white stationary noise. Hence the sum of intercell interference and noise, $v_{l}^{j}$, is stationary. In the case of multiple MS antennas, the total received signal from a BS is

$$
\boldsymbol{y}_{l}=\sum_{j=1}^{J} \boldsymbol{y}_{l}^{j} \otimes \boldsymbol{e}_{j}
$$

where $e_{j}$ is a unit vector of size $J$ with a 1 in position $j$. The total channel is then $\boldsymbol{h}_{l}=\sum_{j=1}^{J} \boldsymbol{h}_{l}^{j} \otimes \boldsymbol{e}_{j}$. 


\section{MAX-SINR RECEIVER STRUCTURE}

As shown in Fig. 2, the receiver is constrained to be a chip rate filter $f$ followed by a descrambler and a correlator with the spreading code of the user of interest, which is here assumed to be user 1 . So the receiver has the same structure as a RAKE receiver, except that the channel matched filter gets replaced by a general filter $f$. If a sparse (pathwise) representation is used for the channel, then the channel matched filter leads to a RAKE structure with one finger per path. In Fig. 2, the operation "S/P" denotes a serial to parallel conversion which stacks the $L$ most recent inputs into a vector. The correlator can also be viewed as a matched filter, matched to the spreading code filter, but here it is simply depicted as an inner product on a downsampled vectorized signal.

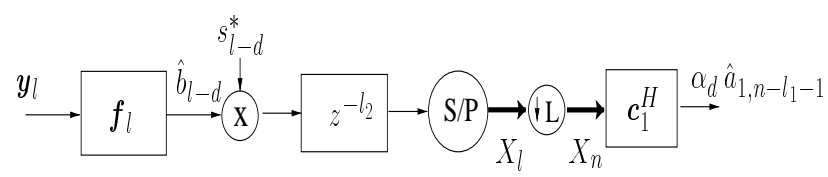

Figure 2. The downlink receiver structure

While the RAKE is one particular instance of the proposed receiver structure, another special case is the equalizer receiver. To describe this case more precisely, let $\boldsymbol{h}(z)=$ $\sum_{l=0}^{N-1} \boldsymbol{h}_{l} z^{-l}$ be the $M J \times 1$ FIR channel transfer function and $f(z)=\sum_{l=0}^{I-1} f_{l} z^{-l}$ the $1 \times M J$ FIR filter transfer function of length $I$ chips. The cascade of channel and filter gives $\boldsymbol{f}(z) \boldsymbol{h}(z)=\sum_{l=0}^{I+N-2} \alpha_{l} z^{-l}=\alpha(z)$. In particular, for a zero-forcing (ZF) equalizer with a delay of $d$ chips, we get $\boldsymbol{f}(z) \boldsymbol{h}(z)=z^{-d}$. In general, we can write for the filter-channel cascade

$$
\mathcal{T}(\boldsymbol{f}) \mathcal{T}(\boldsymbol{h})=\mathcal{T}(\alpha)=\mathcal{T}\left(\alpha_{d}\right)+\mathcal{T}\left(\overline{\alpha_{d}}\right)
$$

where $\mathcal{T}($.$) is a block Toeplitz filtering matrix and$

$$
\begin{aligned}
& \alpha=\left[\alpha_{0} \cdots \alpha_{I+N-2}\right], \alpha_{d}=\left[0 \cdots 0 \alpha_{d} 0 \cdots 0\right]
\end{aligned}
$$

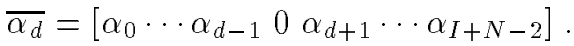

In the noiseless case (and no intercell interference), the use of a ZF equalizer leads to $\overline{\alpha_{d}}=[0 \cdots 0]$ and $\hat{a}_{1, n}=a_{1, n}$ $\left(\alpha_{d}=1\right)$. A RAKE receiver corresponds to $\boldsymbol{f}=\boldsymbol{h}^{H}, \alpha_{d}=$ $\|\boldsymbol{h}\|^{2}, I=N$, where $\boldsymbol{h}=\left[\boldsymbol{h}_{N-1}^{T} \cdots \boldsymbol{h}_{0}^{T}\right]^{T}$.

The analysis done in [3] shows that, due to the orthogonality of the spreading codes and to the i.i.d. character of the scrambler, the SINR at the receiver output, $\Gamma$, is

$\Gamma=\frac{\sigma_{1}^{2}\left|\alpha_{d}\right|^{2}}{f R_{V V} f^{H}+\left.\sigma_{t o t}^{2}|| \overline{\alpha_{d}}\right|^{2}}=\frac{\sigma_{1}^{2}\left|\alpha_{d}\right|^{2}}{f R_{Y Y} f^{H}-\sigma_{t o t}^{2}\left|\alpha_{d}\right|^{2}}$

where $\sigma_{k}^{2}=\mathrm{E}\left|a_{k, n}\right|^{2}, \sigma_{\text {tot }}^{2}=\frac{1}{L} \sum_{k=1}^{K} \sigma_{k}^{2}$ and $R_{Y Y}=R_{V V}+\sigma_{\text {tot }}^{2} \mathcal{T}\left(\boldsymbol{h}^{\prime}\right) \mathcal{T}^{H}\left(\boldsymbol{h}^{\prime}\right)$. The choice for the filter $f$ that leads to maximum receiver output SINR is unique up to a scale factor and can be found as the solution to the following problem

$$
\begin{aligned}
\boldsymbol{f}_{M A X} & =\arg \max _{\boldsymbol{f}: \boldsymbol{f} \boldsymbol{h}=1} \Gamma=\arg \min _{\boldsymbol{f}: \boldsymbol{f} \boldsymbol{h}=1} \boldsymbol{f} R_{Y Y} \boldsymbol{f}^{H} \\
\Rightarrow \boldsymbol{f}_{M A X} & =\left(\boldsymbol{h}^{H} R_{Y Y}^{-1} \boldsymbol{h}\right)^{-1} \boldsymbol{h}^{H} R_{Y Y}^{-1}
\end{aligned}
$$

The maximum SINR becomes $\left(\alpha_{d}^{M A X}=1\right)$

$$
\Gamma_{M A X}=\frac{\sigma_{1}^{2}}{\left(\boldsymbol{h}^{H} R_{Y Y}^{-1} \boldsymbol{h}\right)^{-1}-\sigma_{t o t}^{2}}
$$

As pointed out in [3], this receiver corresponds to the cascade of an (unbiased if $\alpha_{d}=1$ ) MMSE receiver for the desired user's chip sequence, followed by a descrambler and a correlator. In the noiseless case, the MMSE receiver $\boldsymbol{f}_{M A X}$ becomes a $\mathrm{ZF}$ equalizer.

\section{Path-Wise Receiver Structures}

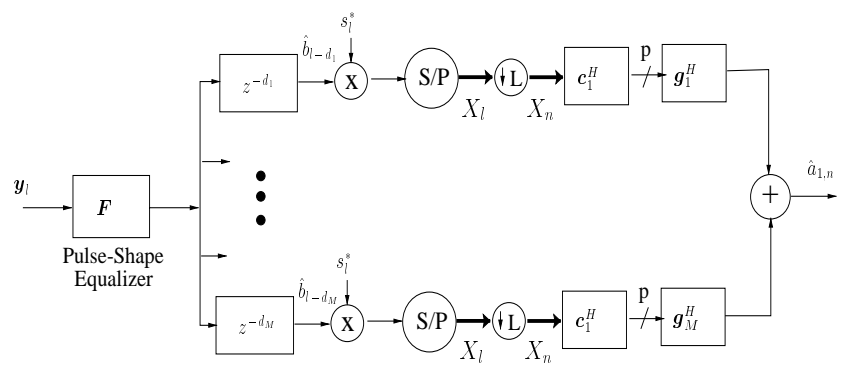

\section{Figure 3. The path-wise equalizer RAKE struc- ture (PWEQ-RAKE)}

The equalizer filter $f_{M A X}$ presented in section 3 replaces at the same time the pulse shape and the channel matched filters, leaving complete freedom to the optimization process. Other possibilities arise when we impose a particular structure on the receiver. We shall here focus on structured equalizers that are the cascade of a short spatiotemporal FIR filter followed by a sparse spatiotemporal filter. The RAKE is a particular instance of this structure, with the FIR filter being the pulse shape matched filter per antenna, and the sparse filter being the 2D matched filter to the $2 \mathrm{D}$ sparse propagation channel. We shall here consider several choices for the short FIR filter, with the sparse filter portion being optimized for max SINR. The receiver depicted in Fig. 3 is one possible instance of the constrained receiver structure considered here.

\subsection{Pulse-Shape MF (GRAKE)}

The pulse shape adopted by the $3 \mathrm{G}$ UMTS norm is a root raised cosine (RRC) with roll-off 0.22 . The short FIR 
filter in this case is simply the pulse shape matched filter, as in the RAKE receiver. However, the sparse propagation channel matched filter keeps its sparse structure, but its coefficients are optimized for maximum SINR. This receiver structure was introduced in [1] as the GRAKE and independently in [4]. To analyze this receiver structure, we can write the overall channel $\boldsymbol{h}$ in (4) as

$$
\boldsymbol{h}=\mathcal{T}^{H}\left(\boldsymbol{p} \otimes I_{J}\right) \boldsymbol{h}_{p r o p}=\mathcal{P}_{s p}^{H} \boldsymbol{h}_{s p}
$$

where $\mathcal{T}^{H}\left(\boldsymbol{p} \otimes I_{J}\right)$ is the convolution matrix of the root raised cosine $p(t)$ and $\boldsymbol{h}_{\text {prop }}$ is the vector of samples of the (sparse) multipath propagation channel (MPC). Due to the sparseness of the MPC (train of pulses), $\mathcal{T}^{H}\left(\boldsymbol{p} \otimes I_{J}\right)$ can be reduced to $\mathcal{P}_{s p}^{H}$ (selected columns) and $\boldsymbol{h}_{p r o p}$ to $\boldsymbol{h}_{s p}$ (the non-zero coefficients of $\boldsymbol{h}_{\text {prop }}$ ). The receiver filter is then factored into a RRC matched filter (represented by a convolution matrix as $\mathcal{T}^{H}\left(\boldsymbol{p} \otimes I_{J}\right)$ or $\left.\mathcal{P}_{s p}\right)$ and an optimized (sparse) filter:

$$
f=g_{\text {prop }} \mathcal{T}\left(\boldsymbol{p} \otimes I_{J}\right)=\boldsymbol{g}_{s p} \mathcal{P}_{s p} .
$$

Optimizing the coefficients of the spatiotemporal (temporally) sparse filter $\boldsymbol{g}_{s p}$ for maximum SINR at the output of the overall receiver, we get for the SINR

$$
\Gamma_{G R A K E}=\frac{\sigma_{1}^{2}}{\left(\boldsymbol{h}^{H} \mathcal{P}_{s p}^{H}\left(\mathcal{P}_{s p} R_{Y Y} \mathcal{P}_{s p}^{H}\right)^{-1} \mathcal{P}_{s p} \boldsymbol{h}\right)^{-1}-\sigma_{t o t}^{2}}
$$

For improved performance, the tap positions in $\boldsymbol{g}_{\text {prop }}$ do not necessarily correspond to the taps in the propagation channel $\boldsymbol{h}_{\text {prop }}$. Also, increasing the number of taps beyond the number of paths (such that more than one tap per path is available) will obviously improve performance, but at the cost of an increase in complexity. In the GRAKE, the channel impulse response as seen at the input of the sparse filter is the channel impulse response filtered by the pulse shape matched filter, or hence the sparse propagation channel filtered by the pulse shape correlation sequence. This sequence would be a delta function if no oversampling would be used. In the case of oversampling however, it leads to a number of nonzero samples. Hence, putting one tap per finger in the sparse filter of the receiver does not appear to be optimal.

\subsection{Path-Wise Equalizer (PWEQRAKE)}

In the unconstrained equalizer-correlator receiver, the optimal equalizer $f_{M A X}$ is essentially a MMSE equalizer $\boldsymbol{h}^{H} R_{Y Y}^{-1}$. Hence, a logical choice for the short FIR filter in the path-wise structured equalizer would be a pathwise equalizer, or hence a MMSE pulse shape equalizer. The resulting receiver is depicted in Fig. 3. One may remark that in that case, the channel as seen at the input of the sparse filter is the cascade of the channel impulse response and the pulse shape equalizer and hence also the cascade of the sparse propagation channel and the equalized pulse shape (cascade of the pulse shape and its MMSE equalizer). This equalized pulse shape should have approximately one significant nonzero coefficient. Hence, the sparse filter with one tap per path appears to be well adapted in this case.

The spatiotemporal FIR MMSE equalizer for the pulse shape is of the form

$$
F=\left(\boldsymbol{p} \otimes I_{J}\right) R_{Y Y}^{-1}
$$

where $R_{Y Y}$ now is the covariance matrix spanning $2 Q+1$ chip periods, and $\boldsymbol{p}$ contains $2 Q+1$ blocks of size $M \times M$, that are filled with the $M$ (oversampling) phases of the pulse shape (matched filter) in the time span $(-Q, Q)$ chip periods. The resulting $F$ contains $M J$ rows, each row corresponding to the pulse shape equalizer for a particular sampling phase and a particular antenna. The receiver filter (overall equalizer) is now again factored into a short FIR filter, being the pulse shape equalizer, and an optimized (sparse) filter:

$$
\boldsymbol{f}=\boldsymbol{g}_{\text {prop }} \mathcal{T}(F)=\boldsymbol{g}_{s p} \mathcal{F}_{s p}
$$

Optimizing the coefficients of the spatiotemporal (temporally) sparse filter $g_{s p}$ for maximum SINR at the output of the overall receiver, we get for the SINR

$$
\Gamma_{P W E Q R A K E}=\frac{\sigma_{1}^{2}}{\left(\boldsymbol{h}^{H} \mathcal{F}_{s p}^{H}\left(\mathcal{F}_{s p} R_{Y Y} \mathcal{F}_{s p}^{H}\right)^{-1} \mathcal{F}_{s p} \boldsymbol{h}\right)^{-1}-\sigma_{\text {tot }}^{2}}
$$

\subsection{Averaged PWEQRAKE (APWEQRAKE)}

The spatiotemporal MMSE equalization of the pulse shape leads to a nonnegligible complexity unless $Q$ is kept very small. To simplify the equalization operation, we can average the RX signal covariance matrix over the antennas to obtain $\bar{R}_{Y Y}$ with which we construct a temporal pulse shape equalizer

$$
\bar{F}=\boldsymbol{p} \bar{R}_{Y Y}^{-1}
$$

We then apply this temporal equalizer to each antenna signal, hence

$$
F=\bar{F} \otimes I_{J}
$$

The SINR for the APWEQRAKE can be obtained by substituting the spatiotemporal pulse shape equalizer $F$ from (14) in (16) by the temporal one in (18). An alternative strategy would be to have an optimized temporal equalizer per antenna instead of an averaged one. 


\section{Simulations}

Simulations were performed to evaluate the output SINR of the various receiver structures as a function of SNR. In the four figures, the SINR curves are averaged over 100 realizations of the channel, which is either "Indoor"-like or "Vehicular"-like. The spreading factor is 32 and 9 users are present in the cell of interest and in the neighboring cell. The SIR between the signals received from the 2 base stations is $0 \mathrm{~dB}$. All users in a cell are at the same power level. The pulse shape equalizer spread $Q$ ("prediction order") is typically 2 for PWEQRAKE and 4 for APWEQRAKE, to have comparable complexity to the GRAKE or RAKE. Typically $\Gamma_{M A X}>\Gamma_{P W E Q R}>\Gamma_{A P W E Q R} \approx \Gamma_{G R}>\Gamma_{R}$ where $R$ stands for $R A K E$. From the previous discussion, one may think that without oversampling, the PWEQRAKE would not be able to improve upon the GRAKE, but the last figure shows the contrary.

\section{References}

[1] T. O. G. Bottomley and Y. Wang. A generalized rake receiver for interference suppression. IEEE Journal Selected Areas Communications, 18:1536-1545, August 2000.

[2] I. Ghauri and D. T. M. Slock. Linear receivers for the DSCDMA downlink exploiting orthogonality of spreading sequences. In Proc. 32nd Asilomar Conf. on Signals, Systems \& Computers, Pacific Grove, CA, November 1998.

[3] M. Lenardi and D. T. M. Slock. A RAKE Receiver with Intracell Interference Cancellation for a DS-CDMA Synchronous Downlink with Orthogonal Codes. In Proc. VTC 2000, Tokyo, Japan, May 2000.

[4] M. Lenardi and D. T. M. Slock. SINR Maximizing Equalizer Receiver for DS-CDMA. In Proc. EUSIPCO 2000, Tampere, Finland, September 2000.

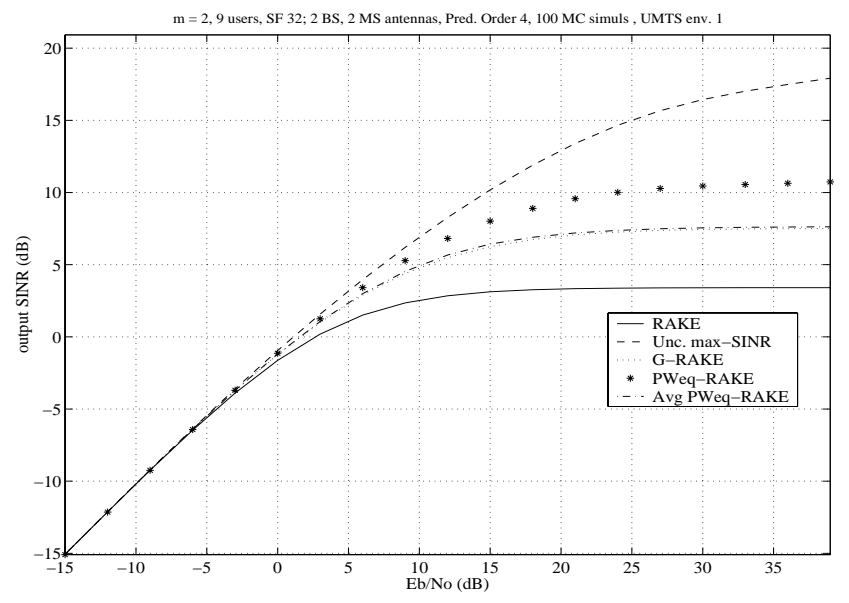

Figure 4. Indoor, $M=2$, J=2.

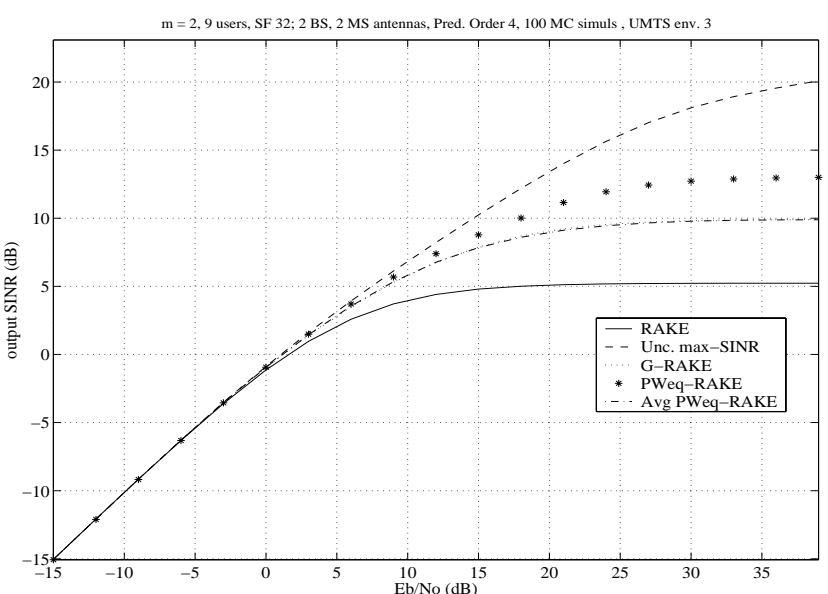

Figure 5. Vehicular, $M=2, \mathrm{~J}=2$.

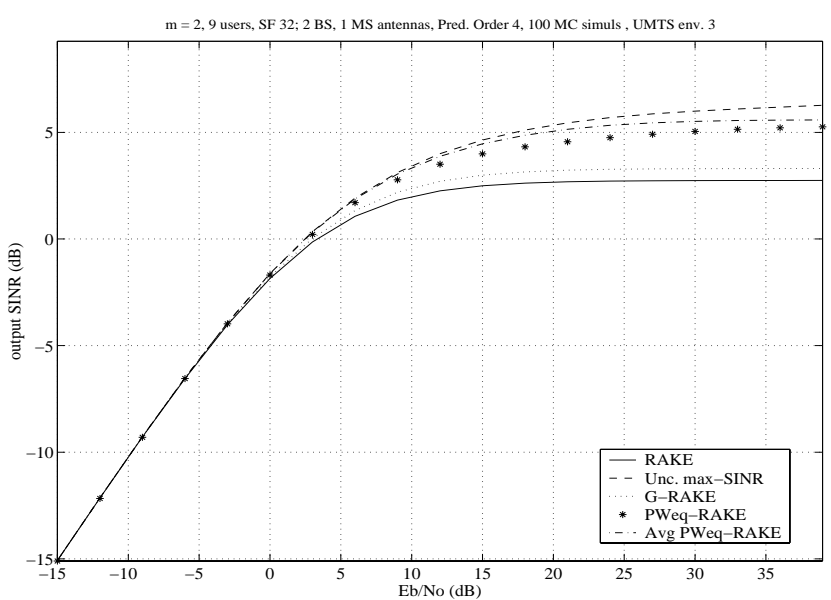

Figure 6. Vehicular, $\mathbf{M = 2 ,} \mathrm{J}=1$.

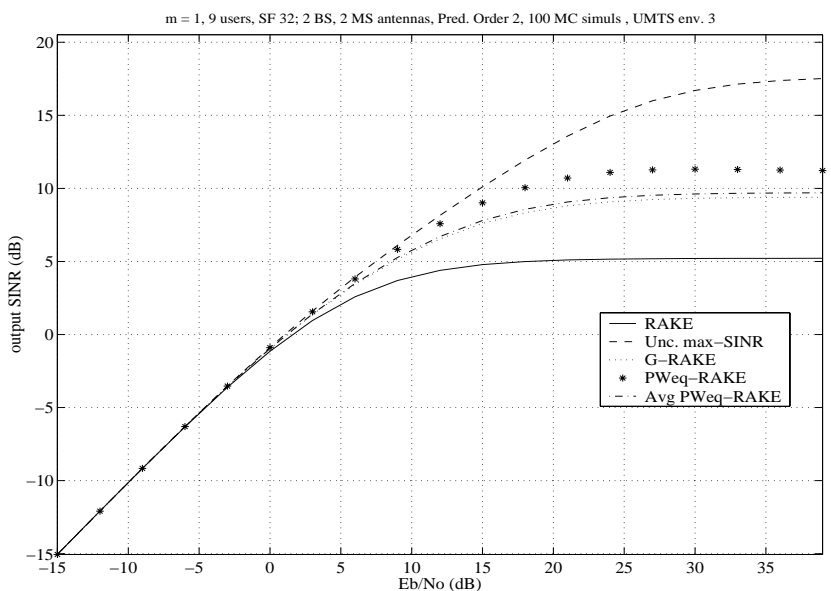

Figure 7. Vehicular, $\mathbf{M = 1}$, J=2. 